Int. J. Dev. Biol. 59: 229-234 (2015)

doi: $10.1387 / \mathrm{ijdb} .140197 \mathrm{LV}$

\title{
Expression patterns of CREB binding protein (CREBBP) and its methylated species during zebrafish development
}

\author{
JULIE BATUT ${ }^{1,2}$, CARINE DUBOÉ ${ }^{1,2}$ and LAURENCE VANDEL ${ }^{*, 1,2}$ \\ ${ }^{1}$ Université de Toulouse, UPS, CBD (Centre de Biologie du Développement) and \\ ${ }^{2}$ CNRS, CBD UMR 5547, Toulouse, France.
}

\begin{abstract}
Proper embryonic development requires a fine-tuned control of gene expression, which is achieved in part through the activity of transcription coactivators or corepressors. The nuclear coactivator cAMP-response element-binding protein (CREB) binding protein (CREBBP or CBP) interacts with numerous transcription factors and thereby plays a key role in various signaling pathways. Interestingly, in cell-based studies CREBBP activity is modulated by post-translational modifications such as methylation on arginine residues which is catalyzed by coactivator-associated arginine methyltransferase 1 (CARM1). However, whether and where CREBBP, and in particular its methylated forms, are expressed during development in vertebrates has not been addressed so far. Here, we analyzed the expression of the two crebbp genes (crebbpa \& crebbpb) during zebrafish development using both RT-qPCR and in situ hybridization. We found that while crebbpa expression is higher in posterior, caudal nascent somites during somitogenesis, crebbpb accumulates in anterior, rostral, and more mature somites. In addition, crebbpa mRNA is enriched in the central myotome at $24 \mathrm{hpf}$ indicating that its expression is spatially and temporally controlled. We next characterized the expression of CREBBP protein from blastula to gastrula stages by immunohistochemistry. We found that while CREBBP is clearly cytoplasmic in the early blastula, it becomes both cytoplasmic and nuclear at $\mathbf{3 0} \%$ epiboly before turning mainly nuclear during gastrulation. Of interest, CREBBP methylated species appear to be mainly nuclear from $30 \%$ epiboly to 6 -somite stage. This suggests that methylation may regulate CREBBP import to the nucleus during zebrafish development and could therefore participate in the control of early developmental processes.
\end{abstract}

KEY WORDS: CREB binding protein, arginine methylation, expression, embryo, zebrafish

During embryonic development, the expression of a given gene has to be temporally and spatially finely controlled. Transcription co-activators such as the highly conserved CAMP response element-binding protein (CREB) binding protein (CREBBP or CBP) and its paralog p300 regulate gene transcription by connecting transcription factors to the basal transcriptional machinery (Vo \& Goodman, 2001). CREBBP co-activates transcription dependent on a large spectrum of transcription factors e.g. pro-proliferative proteins such as c-MYC tumor suppressors and pro-apoptotic proteins like p53, the SMAD proteins but also MyoD (Vo \& Goodman, 2001). Through its protein interaction network, CREBBP is involved in many cellular processes such as DNA repair, cell growth, differentiation and apoptosis (for reviews, (Goodman \& Smolik, 2000; Kalkhoven, 2004)).

Besides its interaction with transcription factors, CREBBP harbors a histone acetyltransferase (HAT) activity (Bannister \& Kouzarides, 1996; Ogryzko et al., 1996) and activates transcription by acetylating histones as well as non-histone proteins on specific lysine residues. Histone acetylation creates docking sites for effectors/partners that will modulate transcription, while acetylation on other proteins such as transcription factors will regulate their DNA binding activity, stability or nuclear import (for a review, (Musselman et al., 2012)). Of interest, we have previously shown

\footnotetext{
Abbreviations used in this paper: $\mathrm{CBP}$ or CREBBP, CREB binding protein; CREB, cAMP responsive element binding protein; CARM1, coactivator-associated arginine methyltransferase 1; PRMT4, protein arginine methyltransferase 4; HAT, histone acetyltransferase; MRF, myogenic regulatory factor; PSM, presomitic mesoderm; ss, somite stage; hpf, hours post-fertilization.
}

\footnotetext{
*Address correspondence to: Laurence Vandel, CBD UMR5547 CNRS, 118 route de Narbonne, 31062 Toulouse cedex, France. Tel: +33-561-558383. Fax: +33-561-556507. E-mail: laurence.vandel@univ-tlse3.fr
}

Supplementary Material (two figures) for this paper is available at: http://dx.doi.org/10.1387/ijdb.ijdb.140197LV

Accepted: 15 January 2015.

ISSN: Online 1696-3547, Print 0214-6282 
that CREBBP is methylated by the coactivator-associated arginine methyltransferase 1 (CARM1) (also called protein arginine methyltransferase 4 (PRMT4)) on four arginine (R) residues in vitro and in cell lines and that CREBBP methylation is important for estrogen receptor-mediated transcription (Ceschin et al., 2011; ChevillardBriet et al., 2002). Furthermore, using highly specific antibodies that recognize each CREBBP methylation site and comparative genome-wide chromatin immunoprecipitation sequencing in a human cancer cell line, we revealed the existence of distinct but overlapping binding site repertoires specific for each methylated CBP species, suggesting that CREBBP methylation by CARM1 specifies distinct estrogen-induced sub-programs (Ceschin et al., 2011). These results raise the possibility that CREBBP methylation may play an important role in the regulation of CREBBP function during embryonic development. Of note, we have previously shown that carm 1 is dynamically expressed during zebrafish development and that CARM1 controls myogenesis in vivo (Batut et al., 2011). However, whether and where CREBBP and in particular its methylated forms are expressed during vertebrate development remain unexplored. Interestingly, CREBBP and its methylation sites are well conserved between human and zebrafish. Hence, zebrafish constitutes a valuable model to assess the expression pattern of CREBBP and its methylated forms during embryogenesis.

Here, we report the expression patterns of zebrafish crebbp transcripts during early development and the expression of CREBBP methylated on two arginines from the blastula to somitogenesis using invaluable monospecific antibodies. Notably, by in situhybridization, we observed an enrichment of crebbpa mRNA expression in the newly formed caudal somites at 18 somite stage (ss) that persisted in the central myotome domain at 24 hours post-fertilization (hpf), whereas crebbpb mRNA accumulated in rostral and more mature somites. Moreover, immunofluorescence experiments revealed that CREBBP subcellular localization is extremely dynamic during early development: while it is cytoplasmic at the oblong stage (3.6 hpf), it becomes both cytoplasmic and nuclear at $30 \%$ epiboly (or $4.75 \mathrm{hpf}$ ) and finally accumulates in the nucleus at the shield stage (or 6 hpf). Furthermore, we investigated the localization of CREBBP methylated on either R714 or R742 with monospecific antibodies and found that these two CREBBP methylated species are exclusively nuclear from $30 \%$ epiboly onwards.

Taken together, our data reveal that beyond a spatial and temporal regulation of crebbpa and crebbpb expression, CREBBP protein subcellular localization is dynamically regulated during early zebrafish development. Moreover, our results suggest a potential role of CREBBP in the cytoplasm at early stages and a role of CREBBP arginine methylation in the regulation of CREBBP import/retention into the nucleus.

\section{Results}

\section{Crebbp transcripts are dynamically expressed during zebraf- ish development}

In zebrafish, two genes encode CREBBP or CBP proteins: crebbpa (also called $C B P-B$ ) and crebbpb (also called $C B P-A$ ). To analyze their relative expression during zebrafish development, real time PCR was performed on RNAs extracted at different embryonic stages (Fig. 1A). We found that both crebbpa and crebbpb were robustly expressed at the 2-cell stage indicating that they are expressed maternally. Strikingly, their expression decreased after the mid-blastula transition to reach their lowest level at the shield stage. While crebbpa expression peaks again at the 16-somite stage (16 ss), crebbpb is less expressed and remains at a plateau from 14 ss to 20 ss. Finally, the expression of both crebbpa and crebbpb decreases at $24 \mathrm{hpf}$ and remains low until $48 \mathrm{hpf}$ (Fig. $1 \mathrm{~A})$. Of note, both genes exhibit a similar expression trend with a peak of expression between 14 ss and 20 ss, a period that corresponds to myogenesis. These data suggest that CREBBP may contribute to this process as previously described for the arginine methyltransferase carm1 (Batut et al., 2011).

We then investigated whether similarly to carm1, crebbp transcripts are regionally expressed during somitogenesis. Somites are transient mesodermal units that form by segmentation of the presomitic mesoderm (PSM) located at the caudal part of the embryo. Each newly formed somite rapidly differentiates into the dorsal dermomyotome from which originate myogenic precursors (Holley, 2007). To further analyze their expression in the somites (where myogenesis takes place), expression of crebbpa and crebbpb was assessed in 10 rostral (anterior) somites versus 10 caudal (posterior) somites of 20 ss embryos. During myogenesis, the early Myogenic Regulatory Factor (MRF) myf5labels the young nascent somites and is therefore strongly expressed in the caudal somites and in the PSM (Ochi \& Westerfield, 2007), while m/c2f encoding the fast myosin light chain skeletal muscle isoform is expressed at around $16 \mathrm{hpf}$ in the first anterior somites formed (Xu et al., 1999). Consistent with these data, RT-qPCR on dissected 20 ss embryos revealed a stronger expression of $m / c 2 f$ in the rostral somites as compared to the caudal somites, while myf5expression was detected only in the caudal somites (Fig. 1B). Interestingly, we found a differential expression of the two crebbp genes: while crebbpa was enriched in myf5-positive nascent somites, crebbpb expression correlated with myf5-negative but $\mathrm{mlc} 2 \mathrm{f}$-positive mature somites (Fig. 1B, caudal and rostral somites respectively). This regionalized expression of crebbpa and crebbpb may reflect a differential function during myogenesis. In particular, the preferential co-expression of crebbpa with the myogenic factor myf5 suggests that crebbpa may play a major role in early myogenesis.

We then analyzed crebbpa and crebbpbexpression pattern by in situ hybridization. In line with the qPCR expression data (Fig. 1A), we observed a maternal expression of crebbpa and crebbpb at the 2-cell stage (Fig. 1C). Both genes appear ubiquitously expressed at the shield and $70 \%$ epiboly (Fig. 1C and Supplementary Fig. 1). However, crebbpa expression becomes spatially restricted in the more caudal regions (trunk and tail somites) at $18 \mathrm{ss}$ with an enriched expression in the PSM (Fig. 1C, asterisk and Fig. 1D upper panels), the structure that gives rise to the somites and strongly expresses myf5 (Holley, 2007). At $24 \mathrm{hpf}$, crebbpa is also expressed in the lens (Fig. $1 \mathrm{C}$ and $1 \mathrm{~F}$, red arrow) and in the retina (Fig. 1F, red arrowhead), the otic vesicle (Fig. 1C, white arrowhead) and weakly in the myotome (Fig. $1 \mathrm{C}$ and $1 \mathrm{~F}$ black arrow). Higher magnification views of the trunk somites at 18 ss showed a graded expression of crebbpaalong the rostral-caudal axis with a stronger expression in caudal somites and in the PSM at 18 ss (Fig. 1D, upper panels). Hence, while crebbpa is ubiquitously expressed from 2 -cell stage to $14 \mathrm{ss}$, its expression is progressively enriched in the caudal somites of the trunk and tail regions at 18 ss (Fig. 1E). In addition, transverse sections at $18 \mathrm{ss}$ and $24 \mathrm{hpf}$ confirmed that crebbpa expression is enriched caudally (Fig. 1F, black arrowhead). Of note, if crebbpb transcript is ubiquitously expressed from the 
shield to $24 \mathrm{hpf}$ (Supplementary Fig. 1), higher magnifications at $18 \mathrm{ss}$ revealed that $c r e b b p b$ is strongly expressed in mature rostral somites (Fig. 1D, lower panels, red asterisk).

All together, our data show that crebbpband crebbpaexpression patterns are complementary at 18 ss with crebbpa and crebbpb being enriched caudally and rostrally, respectively. In addition, these results suggest that crebbpa could be involved in the early step of myogenesis and that both genes could participate in muscle fiber differentiation.

\section{Dynamic regulation of CREBBP subcellular localization during zebrafish early development}

We next investigated by immunohistochemistry the subcellular localization of CREBBPb (or CBP-B), the protein product of crebbpa, after expression of the zygotic genome (512-cell stage or $2.75 \mathrm{hpf}$ in zebrafish) at blastula and gastrula stages. We found that while CREBBPb was mainly cytoplasmic in the early blastula (Fig. 2A, $2 \mathrm{~B}$; oblong and sphere, respectively), it was detected both in the cytoplasm and in the nucleus in the late blastula (Fig. 2C, $30 \%$ epiboly) before accumulating in the nucleus at gastrulation (Figure $2 \mathrm{D}$, shield stage). In conclusion, these data show that CREBBPb subcellular localization is highly dynamic from blastula to gastrula stages (Fig. 2E).

\section{CREBBPb methylated species expression patterns during zebrafish early development}

CREBBP is methylated by CARM1 on several arginine residues in vitro and in human cell lines (Ceschin et al., 2011; ChevillardBriet et al., 2002) but it is currently unknown if and where the different CREBBP methylated species are expressed during development. To address this question, we took advantage of monospecific antibodies that were raised against human CREBBP methylated by CARM1 at arginines 714 and 742 (noted R714me2a and R742me2a). Importantly, the specificity of these antibodies was extensively validated in human cells as well as in zebrafish in which CREBBP methylation sites are conserved ((Ceschin et al., 2011), Fig. 3A and Supplementary Fig. 2). In particular, using morpholino (Mo) against crebbpa (CREBBPb or CBP-B) we
A

crebbp expression during development
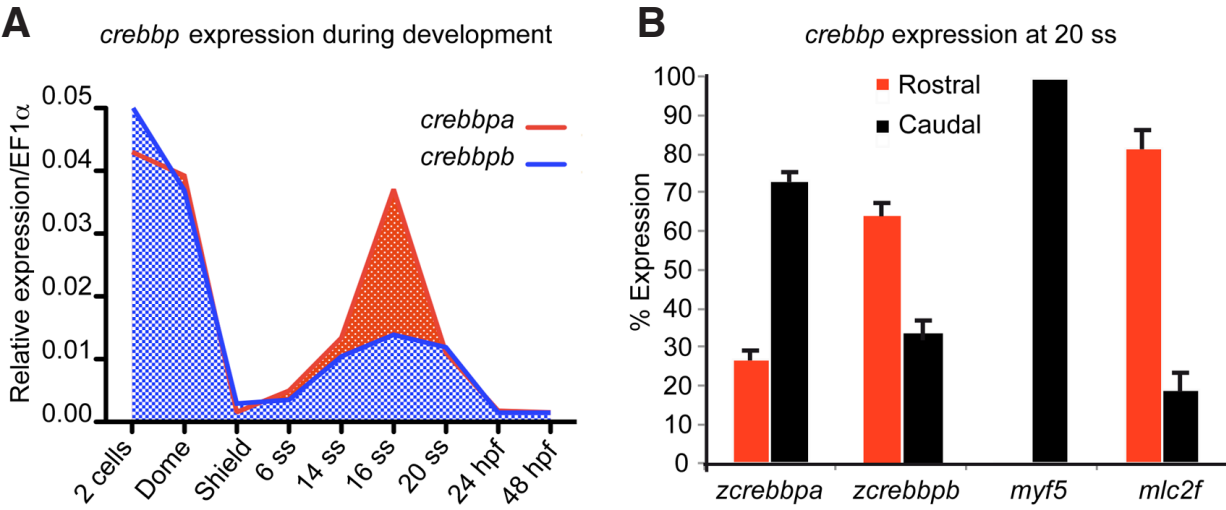

C

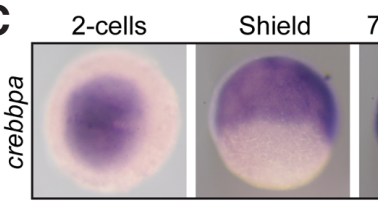

$70 \%$ Epiboly

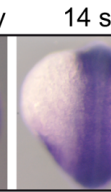

D
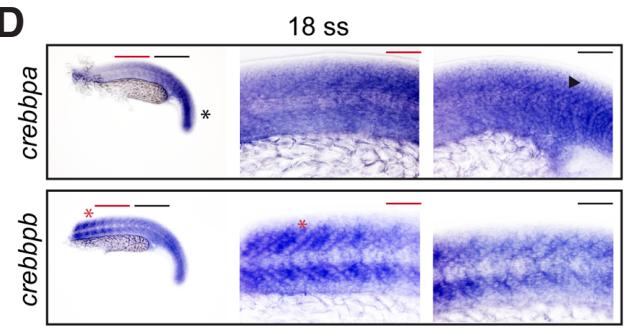

F

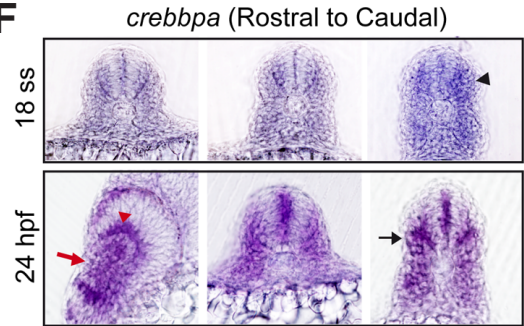

$18 \mathrm{ss}$

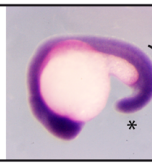

E

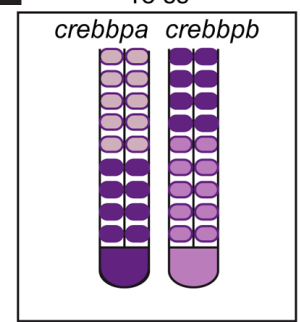

$24 \mathrm{hpf}$

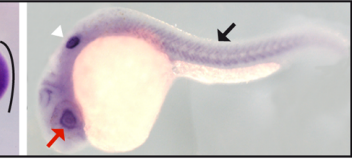


found that antibodies recognizing $R 714$ me2a and $R 742 m e 2 a$ of human CREBBP were specific for CREBBPb as revealed by immunofluorescence (Supplementary Figure 2A, 2B). As CREBBPb could not be detected by immunofluorescence after $1 \mathrm{ss}$ (data not shown), western blots were performed on CREBBPb- or control morpholino-injected embryos to confirm that anti-CREBBP antibody was specifically recognizing CREBBPb. Consistently, CREBBPb protein expression was extinguished following CREBBPb-MO injection (Supplementary Fig. 2C).

When comparing by immunohistochemistry the subcellular localization of CREBBPb and its methylated forms in the late blastula (30\% epiboly), we found that while CREBBPb was expressed both in the cytoplasm and in the nucleus, CREBBPb methylated forms were enriched in the nucleus (Fig. 3B). In addition, both CREBBPb

A

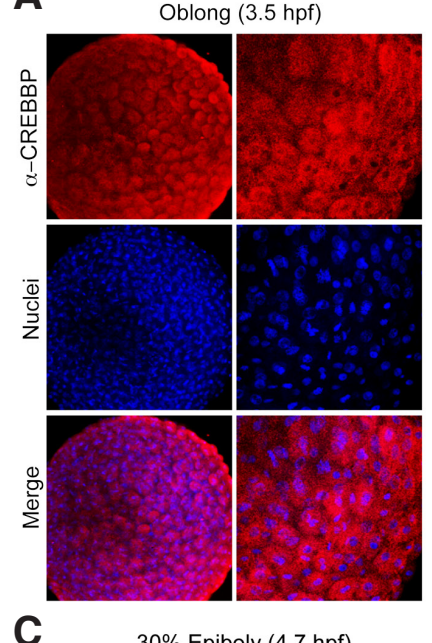

C

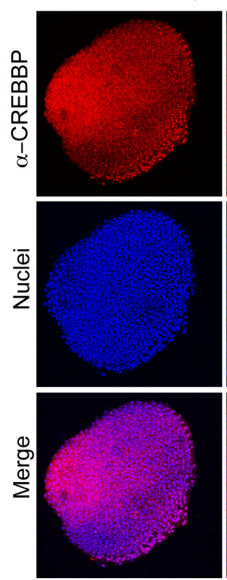

E
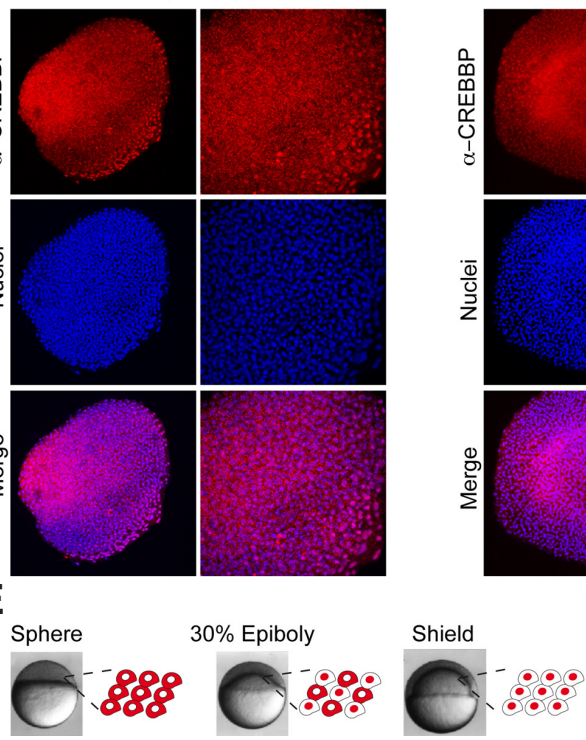

Fig. 2. CREBBP protein expression patterns from blastula to gastrula. (A-D) Immunohistochemistry with CREBBP specific antibody at(A) oblong, (B) sphere, (C) $30 \%$ epiboly and (D) shield stages. Nuclei are visualized in blue with TO-PRO3 staining and a merged picture is shown. (E) Schematic illustration of CREBBP subcellular localization at these stages, cells are shown with CREBBP labeled in red. methylated species remained nuclear at least until somitogenesis (6 ss) (Fig. 3C). These results suggest that CREBBP methylation could regulate CREBBP nuclear accumulation.

\section{Discussion}

The transcriptional co-activator CREBBP has been involved in many biological functions due to its HAT activity and to its interaction with numerous transcription factors. CREBBP plays a key role in transcriptional control by modifying histones but also non histone proteins. Hence, CREBBP controls many major signaling pathways, such as TGF $\beta$, nuclear receptors, Hh and Wnt (Goodman \& Smolik, 2000). These signaling pathways cooperate during embryonic development to achieve the formation of a proper body axis. In addition, CREBBP can be post-translationally modified and these modifications regulate CREBBP function. In particular, CREBBP is methylated on specific arginine residues $(R)$ by CARM1 (Chevillard-Briet et al., 2002; Ceschin et al., 2011) and CARM1 has been found to control myogenesis and muscle fibers differentiation during zebrafish embryogenesis (Batut et al., 2011). The fact that crebbpa transcript was enriched in the caudal myf5positive somites and in the presomitic mesoderm could reflect its function in the control of early somitogenesis/myogenesis events such as somite formation. The caudal expression of crebbpa could also indicate that its transcriptional regulation depends on Notch, Wnt/ $\beta$-catenin, Retinoic Acid (RA) or fibroblast growth factor (FGF) signaling pathways, which are known to control somitogenesis (Pourquie, 2011). Thus, CREBBP could regulate transcriptional programs required for proper somite/muscle development downstream of these major signaling pathways. We also found that crebbp transcripts are widely expressed in the head area from 18 ss to $24 \mathrm{hpf}$. Of interest, at $24 \mathrm{hpf}, \mathrm{crebbpb}$ expression is enriched in the lens and in the otic vesicle, two cranial sensory organs (for a review, Schlosser, 2014). Among others, FGF signaling pathway is required for both lens and otic placode development (Schlosser, 2014). Hence, it will be of interest to analyze whether CREBBP fine-tunes this pathway to control proper somite/muscle, lens and otic vesicle development.

Of note, a systematic analysis of the dynamic spatiotemporal expression of all nuclear receptor transcripts as well as their main transcriptional coregulators (including CREBBP) by whole-mount in situ hybridization has been previously conducted during zebrafish development. This study revealed that crebbpa and crebbpb profiles were ubiquitous during development and suggested that the tissue specificity of hormone action was primarily conferred by the localized expression of the receptors (i.e transcription factors)(Bertrand et al., 2007). However, this study was performed on a large set of nuclear receptors and the expression pattern of crebbpa and crebbpb was not examined in detail. Here, we found that the expression of these two genes is distinct and is spatiotemporally regulated.

In addition, we provide the first description of the expression and the subcellular localization of CREBBP and in particular of its methylated species in vivo during early development. Our data indicating that CREBBP was cytoplasmic at early zygotic expression stages (such as blastula stage) was unexpected for a transcriptional co-activator. Of interest, CREBBP localizes both to the cytoplasm and the nucleus when epiboly begins and accumulates in the nucleus at the start of gastrulation. The nuclear accumulation 
A $\begin{array}{cc} & \text { R714 } \\ \text { hCREBBP } & \text { PLPLPVNRMQVSQGM } \\ \text { CREBBPb } & \text { PNQI-LNRMQVSQGM } \\ & \text { R742 } \\ \text { hCREBBP } & \text { PQAPMGPRAASPMNH } \\ \text { CREBBPb } & \text { SQAPMGTRAASPIGP }\end{array}$

B

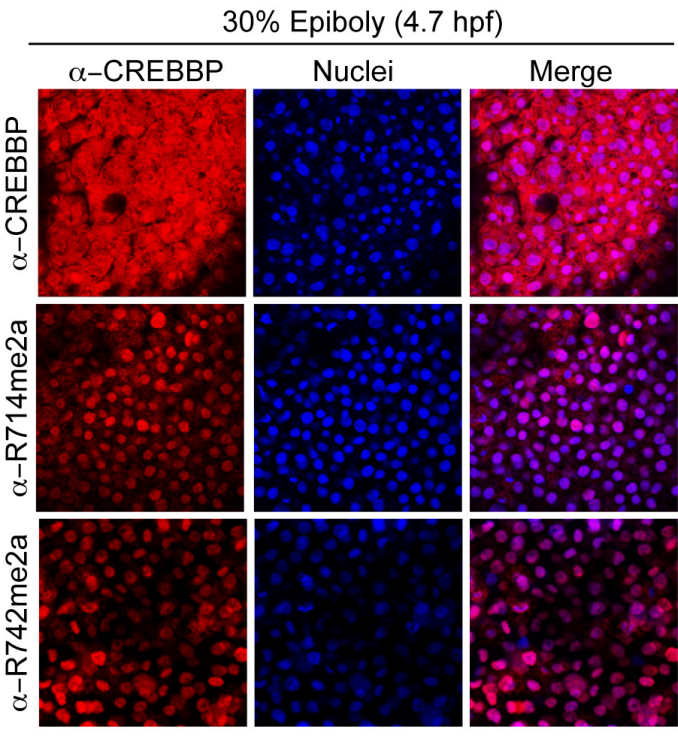

B'

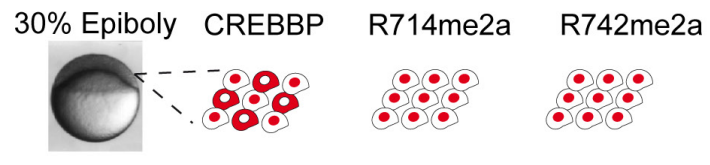

C

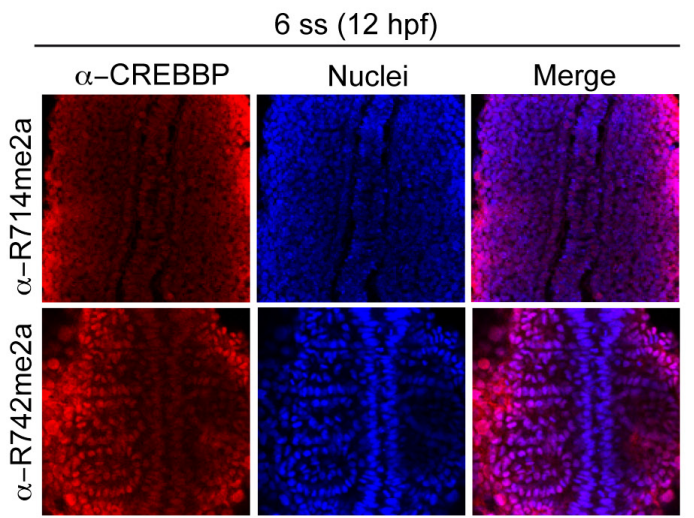

C'

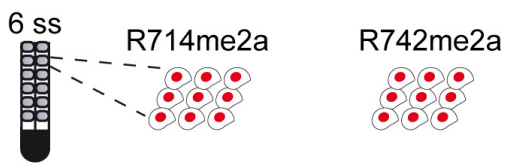

Fig. 3. CREBBP-methylated species expression pattern at late blastula (30\% epiboly) and during somitogenesis (6 ss). (A) Alignment of the epitopes of human CREBBP ( $h C R E B B P$ ) arginines $R 714$ and $R 742$ with the corresponding sequences of zebrafish crebbpa coding protein (CREBBPb or CBP-B). (B,C) Immunohistochemistry with CREBBP and CREBBPmethylated specific antibody as indicated at (B) 30\% epiboly and (C) 6 ss. Nuclei are visualized in blue and a merged picture is shown. (B', $\left.\mathbf{C}^{\prime}\right)$ Schematic illustration of subcellular localization of CREBBP and CREBBPmethylated proteins, with CREBBP labeled in red. of CREBBP at epiboly and gastrulation stages suggests that the movements occurring at these stages require CREBBP to regulate gene transcription. In contrast, in the early blastula, our data indicate that CREBBP is unlikely to function as a nuclear transcription co-activator but may exert a critical role in the cytoplasm. In this view, it would be of great interest to unravel CREBBP acetylation targets in the cytoplasm and to characterize how its import to the nucleus and/or retention to the cytoplasm are regulated.

Interestingly, we found that the two CREBBP methylated species that we analyzed are nuclear at $30 \%$ epiboly, while "total" CREBBP protein is observed both in the nucleus and in the cytoplasm. This indicates that at this stage, nuclear CREBBP is mostly methylated, while cytoplasmic CREBBP corresponds to non-methylated forms on these sites. Hence, methylated CREBBP species are likely to participate in controlling gene expression during epiboly and gastrulation. Moreover, the fact that CREBBP methylated forms are mainly nuclear suggests that methylation may regulate CREBBP import to the nucleus. Finally, we found that during early somitogenesis (6 ss) CREBBP methylated forms on R714me2a and $R 742 m e 2 a$ are present in the nucleus of the somites. Since CARM1 has been found to participate in myogenesis and muscle fibers differentiation (Batut et al., 2011), our observations suggest that CREBBP methylated on these sites could control the gene networks that drive somitogenesis/myogenesis. Indeed, CREBBP methylation has been shown to enhance CREBBP HAT activity and to regulate CREBBP target gene repertoire (Ceschin et al., 2011). Along that line, it would be interesting to analyze the recruitment of CREBBP and its methylated species on chromatin to identify the genes that are regulated by CREBBP and/or methylated CREBBP at these stages.

In conclusion, our results revealed a spatial and temporal regulation of CREBBP expression and methylation during development as well as a probable cytoplasmic function for CREBBP. Deciphering the function(s) of CREBBP in the cytoplasm and the role of its methylation during development would be an exciting and promising opening in the field of epigenetics in development.

\section{Materials and Methods}

\section{Ethics Statement and Embryos}

All embryos were handled according to relevant national and international guidelines. French veterinary service approved the protocols in this study, with approval ID: A-31-555-01. Zebrafish were raised according to standard procedures (Westerfield, 1995).

\section{RNA extraction and reverse transcription}

Total RNAs were extracted from 25 embryos at the indicated stages with the Aurum total RNA mini kit (Bio-Rad), and reverse-transcribed with the iScript cDNA Synthesis Kit (Bio-Rad) according to the supplier's instructions. Note that at 20 ss embryos were sectioned in two parts, the first 10 somites (rostral somites) and the remaining 10 somites (caudal somites), which were subsequently subjected to RNA extraction and reverse transcription independently.

\section{Whole-mount in situ hybridization, immunostaining and image ac- quisition}

Whole-mount in situ hybridization and antibody staining were performed according to standard protocols. In situ hybridization was performed with digoxigenin-labeled RNA probe transcribed from plasmid containing crebbpa and crebbpb probes. crebbpaanti-sense probe (crebbpa-727) was obtained by cloning a $727 \mathrm{bp}$ PCR amplicon from cDNA of 25 embryos at $24 \mathrm{hpf}$ 
with the primers F- 5'-CTCTACAGTAGGCAATCTGAGTAAC-3' and R-5'CATGTTGACGTTCATGGTGTTGGTG-3' into pGEM-TEasy vector (Promega). The resulting vector, pGEM-TEasy-CREBBP-727 was sequenced and was found to be identical to crebbpa-201 (ENSDART00000087250). crebbpaanti-sense probe was generated by linearizing the plasmid with Apal followed by transcription with SP6 polymerase. crebbpb anti-sense probe (exon 14) was obtained by cloning a $330 \mathrm{bp} \mathrm{PCR}$ amplicon from cDNA of 25 embryos at 14 ss with the primers F-5'-CAGCAGCAGCAGAACGCTAAC-3' and R- 5'-CGGAGTGCTTGGCTGCTGCG-3' into pGEM-TEasy. The resulting vector, pGEM-TEasy-crebbpb was sequenced and was identical to crebbpb-201 (ENSDART00000091873) exon 14 (ENSDARE00000948500). $c r e b b p b$ anti-sense probe was generated by linearizing the plasmid with Sacll followed by transcription with SP6 polymerase.

For cross-sections, embryos were embedded in gelatine/albumin after whole-mount in situ hybridization and $30 \mu \mathrm{m}$ sections were performed using a Leica vibratome (VT 1000S).

Zebrafish protein crebbpa (also called CREBBPb or CBP-B) corresponds to protein ID ENSDARP00000081684, while crebbpb protein ID (also called CREBBPa or CBP-A) corresponds to ENSDARP00000086306.

For immunohistochemistry, the following antibodies were used: antiCREBBP (A22, from Santa Cruz Biotechnology), anti-CREBBP ${ }_{\text {R714me2a }}$ and anti-CREBBP ${ }_{\mathrm{R742me2a}}$ (Ceschin et al., 2011), together with appropriate Alexa Fluor-conjugated secondary antibodies (Molecular Probes). Nuclei were stained with TO-PRO3 (Molecular Probes) according to the manufacturer's protocol. Embryoswere dissected, flat-mounted in $70 \%$ glycerol or mounted in Mowiol and images were recorded on a microscope (NIKON Eclipse 80i) using a 20x Plan Apo na 0.5 or a $40 x$ plan Apo na 1 with the NISelement AR 2.30 software, or on a confocal microscope (TCS SP5, Leica Microsystems) with a 20x Plan Apo na 0.7 objective (zoom X4) using the scanner resonant mode. Confocal images are stacks of the anterior somites of 6 ss embryos, animal region of $30 \%$ epiboly embryos or at the indicated stages in figure legends. For whole embryos, imaging was performed using a stereomicroscope (Leica MZ FL III) with the ACT-1C software.

\section{Quantitative PCR}

Q-PCR analyzes were performed on MylQ device (Bio-Rad) with the SsoFast EvaGreen Supermix (Bio-Rad), according to the manufacturer's instructions. All experiments included standard curves. Samples were normalized to EF1 mRNAcopies. Primer sequences were: crebbpa F-5'-CGAAAAGTGGAAGGGGACAT-3', R-5'-TTCTCTTCCAGCTCTTTCTGG-3'; crebbpbF5'-CAGGTTCCTCAAGGGATGG-3'; R-5'-CCATCATGGCTTGAGCTTG-3'; myf5 F- 5'-GAGAGCATGGTTGACTGCAA-3', R -5'-GAATCACTTCCGGTTGGAGA-3'; m/c2fF-5'-ACGACCTTAGGGACGTGTTG-3', R- 5'-CTTGAAGGCAGACACGATGA-3'; EF1 $\alpha$ F- 5'-GATGCACCACGAGTCTCTGA-3', R- 5'-TGATGACCTGAGCGTTGAAG-3'.

\section{Acknowledgements}

We would like to thank Dr. Hinrich Gronemeyer and Michèle Lieb for providing antibodies against methylated CREBBP; Dr. Elise Cau, Dr. Cathy Danesin and Dr. Fernanda Bajanca for critical reading of the manuscript;
Céline Gohory, Vanessa Soldan and Aurélie Le Ru for initialin situ hybridizations and imaging; the IBiSA Toulouse Imaging Platform and Brice Ronsin for microscopy assistance; members of the CBD for helpful discussions and Stéphane Relexans for taking care of the fishes. This work was supported by an "Action Thématique et Incitative sur Programme" from the Centre National de la Recherche Scientifique and from the Fondation ARC pour la Recherche contre le Cancer to L.V and by the Association Française contre les Myopathies to J.B.

\section{References}

BANNISTER AJ, KOUZARIDES T (1996). The CBP co-activator is a histone acetyltransferase. Nature 384: 641-643

BATUT J, DUBOE C, VANDEL L (2011). The methyltransferases PRMT4/CARM1 and PRMT5 control differentially myogenesis in zebrafish. PLos One 6: e25427

BERTRAND S, THISSE B, TAVARES R, SACHS L, CHAUMOT A, BARDET PL, ESCRIVA H, DUFFRAISSE M, MARCHAND O, SAFI R, THISSE C, LAUDET V (2007). Unexpected novel relational links uncovered by extensive developmental profiling of nuclear receptor expression. PLoS Genet 3: e188

CESCHIN DG, WALIA M, WENK SS, DUBOE C, GAUDON C, XIAO Y, FAUQUIER L, SANKAR M, VANDEL L, GRONEMEYER H (2011). Methylation specifies distinct estrogen-induced binding site repertoires of CBP to chromatin. Genes Dev 25: $1132-1146$

CHEVILLARD-BRIETM, TROUCHED, VANDELL(2002). Control of CBP co-activating activity by arginine methylation. Embo $\mathrm{J} 21: 5457-5466$

GOODMAN RH, SMOLIK S (2000). CBP/p300 in cell growth, transformation, and development. Genes Dev 14: 1553-1577

HOLLEY SA (2007). The genetics and embryology of zebrafish metamerism. Dev Dyn 236: 1422-1449

KALKHOVEN E (2004). CBP and p300: HATs for different occasions. Biochem Pharmacol 68: 1145-1155

MUSSELMAN CA, LALONDE ME, COTEJ, KUTATELADZE TG (2012). Perceiving the epigenetic landscape through histone readers. Nat Struct Mol Biol 19: 1218-1227

$\mathrm{OCHI} \mathrm{H}$, WESTERFIELD M (2007) Signaling networks that regulate muscle development: lessons from zebrafish. Dev Growth Differ 49: 1-11

OGRYZKO VV, SCHILTZ RL, RUSSANOVA V, HOWARD BH, NAKATANI Y (1996) The transcriptional coactivators p300 and CBP are histone acetyltransferases. Cell 87: 953-959

POURQUIE O (2011). Vertebrate segmentation: from cyclic gene networks to scoliosis. Cell 145: 650-663

SCHLOSSER G (2014). Early embryonic spécification of vertebrate cranial placodes. Wiley Interdiscip Rev Dev Biol 3: 349-363

VO N, GOODMAN RH (2001). CREB-binding protein and p300 in transcriptional regulation. J Biol Chem 276: 13505-13508

WESTERFIELD M (1995). A Guide for the Laboratory Use of Zefrafish (Danio rerio) OR: University of Oregon Press: Eugene.

XU Y, HE J, TIAN HL, CHAN CH, LIAO J, YAN T, LAM TJ, GONG Z (1999). Fast skeletal muscle-specific expression of a zebrafish myosin light chain 2 gene and characterization of its promoter by direct injection into skeletal muscle. DNA Cell Biol 18: 85-95 


\section{Further Related Reading, published previously in the Int. J. Dev. Biol.}

Visualization, characterization and modulation of calcium signaling during the development of slow muscle cells in intact zebrafish embryos

Chris Y. Cheung, Sarah E. Webb, Donald R. Love and Andrew L. Miller

Int. J. Dev. Biol. (2011) 55: 153-174

http://dx.doi.org/10.1387/ijdb.103160cc

Hematopoietic stem cell emergence in the conceptus and the role of Runx1

Gemma Swiers, Marella de Bruijn and Nancy A. Speck

Int. J. Dev. Biol. (2010) 54: 1151-1163

http://dx.doi.org/10.1387/ijdb.103106gs

Histone deacetylase 1 and 2-controlled embryonic development and cell differentiation Reinhard Brunmeir, Sabine Lagger and Christian Seiser

Int. J. Dev. Biol. (2009) 53: 275-289

http://dx.doi.org/10.1387/ijdb.082649rb

Chromatin states of developmentally-regulated genes revealed by DNA and histone methylation patterns in zebrafish embryos

Leif C. Lindeman, Cecilia L. Winata, Håvard Aanes, Sinnakaruppan Mathavan, Peter Aleström and Philippe Collas

Int. J. Dev. Biol. 54: 803 - 813 (2010)

http://dx.doi.org/10.1387/ijdb.103081I

Developmentally regulated expression of the transcriptional cofactors/histone acetyltransferases CBP and p300 during mouse embryogenesis

A Partanen, J Motoyama and C C Hui

Int. J. Dev. Biol. 43: 487 - 494 (1999)

http://dx.doi.org/10.1387/ijdb.103081II

5 yr ISI Impact Factor $(2011)=2.959$
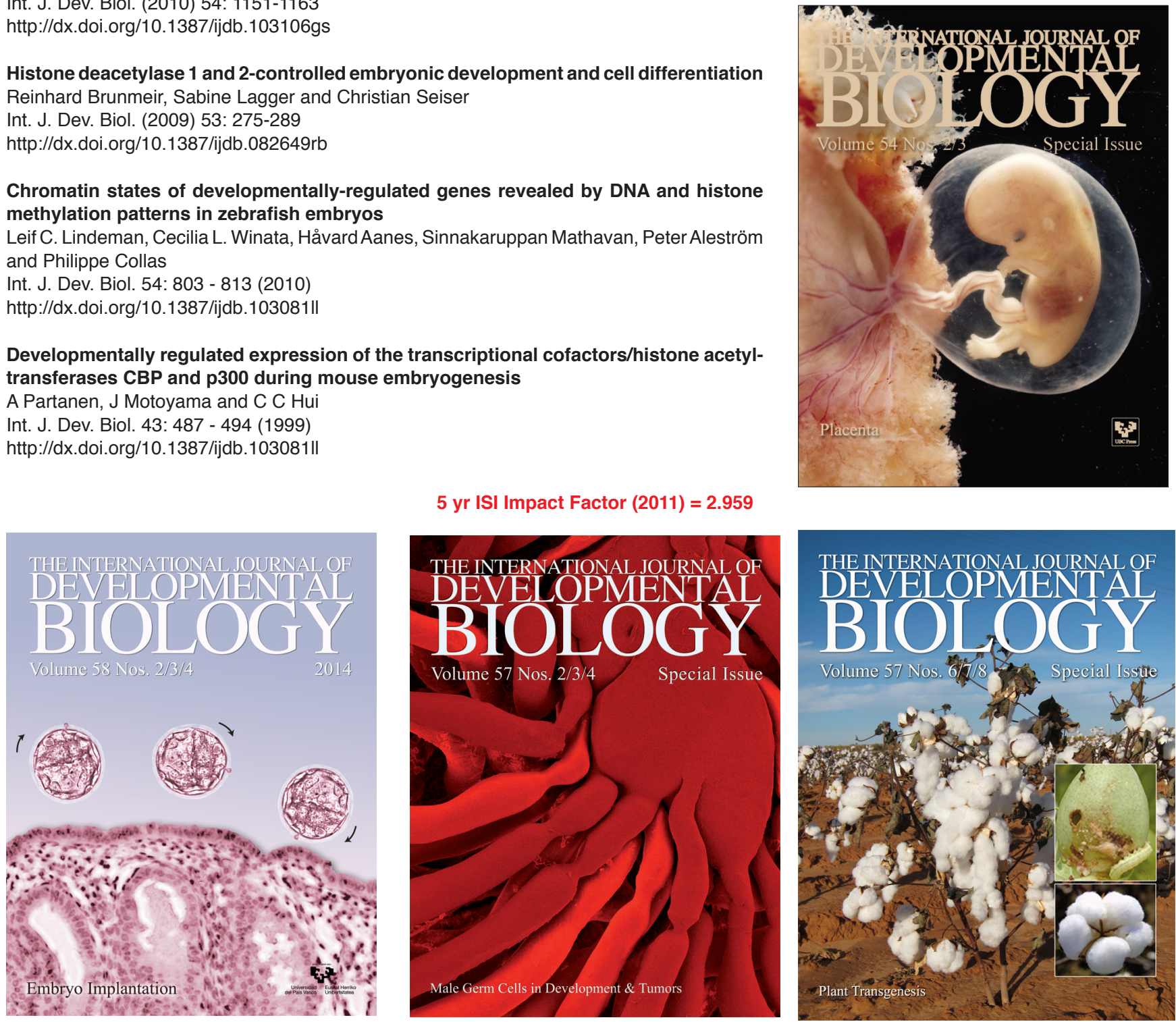

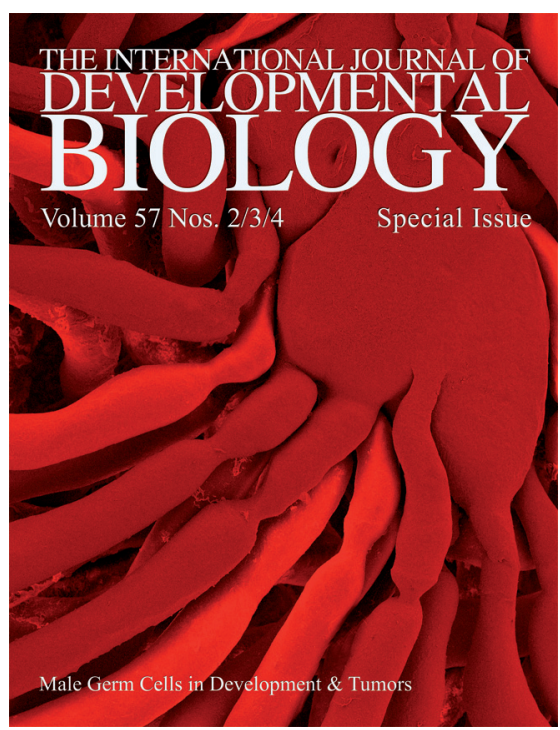

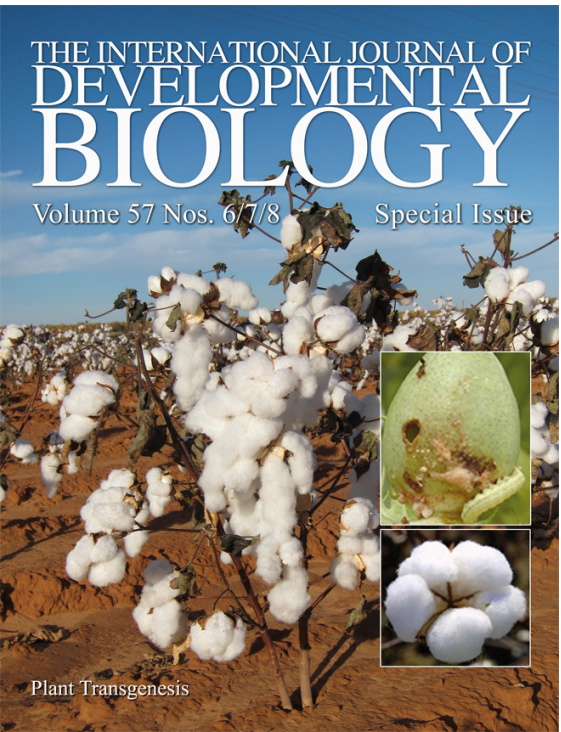

